\title{
An interview with Elisabeth Hsu on Plants, Health and Healing: On the Interface of Ethnobotany and Medical Anthropology
}

\author{
Sofia A. Vougioukalou \\ Author address: King's College London, Department of Primary Care and Public Health Sciences, 42 Weston street, London \\ SE1 3QD \\ sofia.vougioukalou@kcl.ac.uk
}

Received: September 17, 2012

Volume: 3:91-95

Published: December 29, 2012

(C) 2012 Society of Ethnobiology

In this interview Professor Elisabeth Hsu discusses ethnobiology as an interdisciplinary science and introduces the book she co-edited with Dr. Stephen Harris Plants, Health and Healing: On the Interface of Ethnobotany and Medical Anthropology (Hsu and Harris 2010). She discusses epistemological contradictions between biologically and anthropologically orientated ethnobiological studies and argues for a more anthropologically grounded and methodologically rigorous discipline. The interview took place at the School of Anthropology and Conservation at the University of Kent in the UK in February 2011.

The anti-malarial qinghaosu (Artemisinin) captured Elisabeth's attention when she was doing fieldwork in the early 2000s on Chinese medicine in East Africa, as qinghaosu constituted over $50 \%$ of the over-thecounter transactions in the private Chinese medical clinics she visited, which mostly catered to the Swahili -speaking local clientele. Dawa ya Kichina, "the medicine from China", was in this case a purified chemical substance that Chinese scientists had extracted, isolated and chemically identified in the 1970s and 1980s from a traditional Chinese medical plant. It was a Western medical drug, a molecule extracted from the plant materials of Artemisia annua L., which, as Elisabeth demonstrates in her article in this book, since antiquity had been praised for its parasite-killing and wound-healing properties and since medieval times for its use against intermittent fevers. At the time, thousands of articles had been published on the unusual structure of the molecule, its pharmacokinetics, its toxicity and its clinical effects, but not a single one on the history of qinghao in the Chinese medical literature. This prompted Elisabeth into launching a seminar series out of which grew this book project.
Dr Sofia A. Vougioukalou (SAV): What were your incentives in compiling this volume and selecting the contributions that feature in it?

Professor Elisabeth Hsu (EH): This is a coauthored book with Dr. Stephen Harris who is the curator of the herbarium at the University of Oxford and who has also done very interesting research into the history of botany. The volume is based on the Medical Anthropology Research Seminar series 'Ethnobotany, Health and Healing' which we held in winter 2004 at the Institute of Social and Cultural Anthropology at the University of Oxford, shortly after setting up our masters course which aims to combine social and biological anthropological approaches to medical anthropology. As Professor Roy Ellen noted in the 9th International Congress of Ethnobiology in June in the same year at the University of Kent, although $70 \%$ of humankind depends on medicinal plant preparations, there is actually very little academic research on this subject. His 'Ethnobiology and Sciences of Humankind' keynote therefore makes our project all the more timely and relevant.

The book is based on the presentations given in the seminar series. The emphasis was on thick description and ethnography. Wenzel Geissler and Ruth Prince show how the Luo of Kenya use plants to enhance children's growth and rather than focusing on how a particular chemical compound of a particular species is used therapeutically, they highlight how plants are an integral aspect of social practices of belonging, child care and preventive health; and those are fun and playful! Francoise Barbira-Freedman's piece asks why in the Upper Amazon shamans are mostly men. She shows that plants are gendered and that shamans have to seduce them to engage them in the healing 
process. We wanted to be broad so we also included clinical medical, botanical and historical research.

SAV: One of the book's strengths, that highlights its ability to cross disciplinary boundaries, is that its editors are a medical anthropologist and a botanist. For botanists, the core of ethnobotanical research is the accurate identification of medicinal plant species. However, this is not regarded a priority by medical anthropologists and is frequently ignored. How did your partnership work?

EH: Stephen Harris and I are based in the same university and in the same college, Green Templeton College. In the college we are encouraged to undertake joint projects. Sir John Grimley Evans is an Emeritus Fellow at the same college, a distinguished gerontologist, who is rather sceptical about the alleged memory-enhancing effects of Gingko biloba leaf extracts for Alzheimer's patients. He provides a really

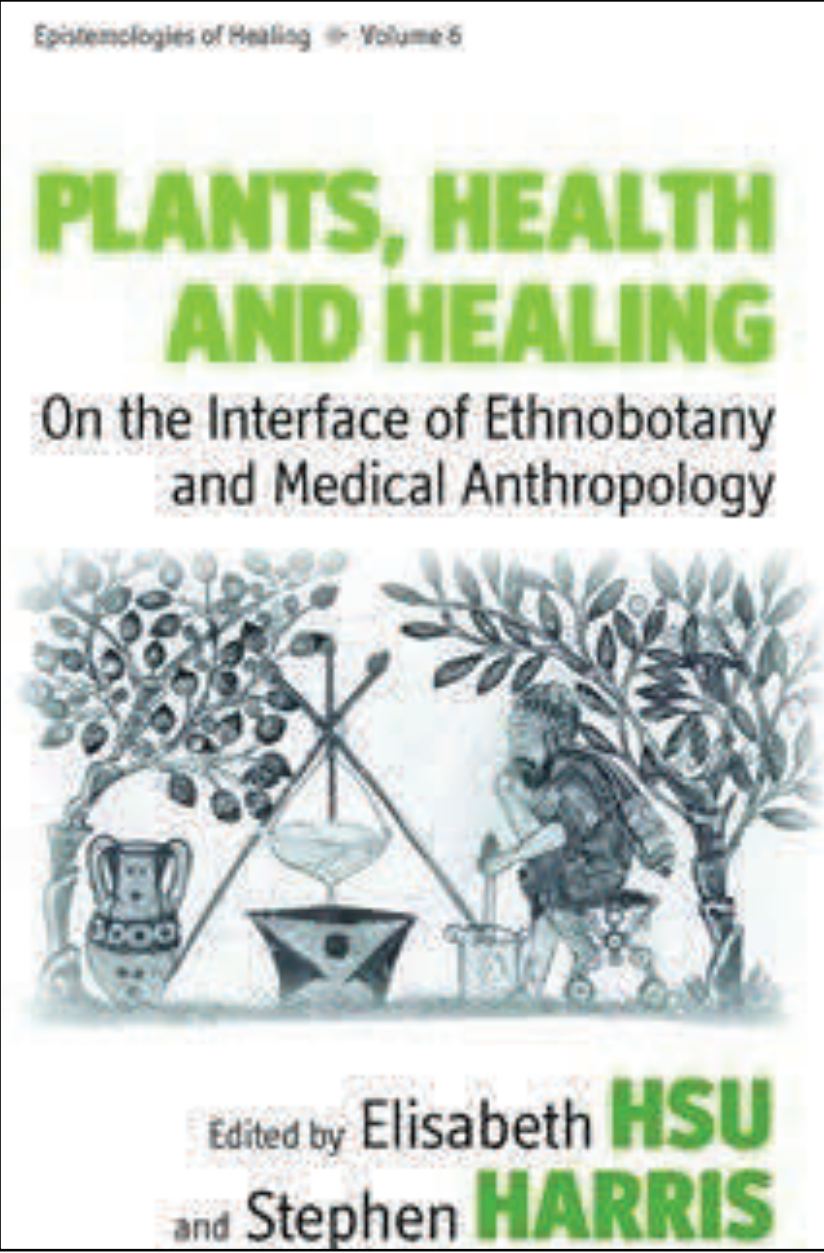

Figure 1. Book cover for Plants, Health and Healing: On the Interface of Ethnobotany and Medical An- succinct appraisal of RCTs in general, and shows that there are none to prove it. If these leaf extracts have any effect, it is indirect: his hunch is that they modulate mood and reduce depression and thereby may enhance people's performance in memory tests. The identification of medicinal plant species indeed is very important but there are other important issues in the way plants are used that are neglected by ethnobotanists. For example, Geissler and Prince show that for the plants used externally in the baths that enhanced Luo childrens' growth, it was important that the plants used grew on the land that belonged to the clan. The people connected to their land through the plants. The authors also had the plants botanically identified by Stephen Harris. So one can do both, write an ethnographic narrative to emphasise what is important for the people and also in the footnotes provide the identification of the species and the range of plant species used.

SAV: This is a great example of interdisciplinary work actually working, especially bearing in mind the long history of animosity between anthropologists and biologists that study the same topic and tend to disregard each other. It shows the benefits that come out when people actually collaborate.

EH: It is important when we collaborate that we actually are willing to learn a bit about both languages, to be multi-lingual does service to both audiences. For example, Harris mentions plant species names in the text and plant appearances in a footnote. Whereas Geissler and Princes' plant species names feature in footnotes. This is one way in which different disciplines can collaborate and still maintain their focus of interest.

SAV: Your introduction presents an astute critique of medicinal ethnobotany. What in your view has ethnobotany in the UK been missing so far?

EH: I only know a little bit about medicinal ethnobotany and it evidently has developed very much in a cognitivist direction and not taken account of recent developments in medical anthropology. A lot of the writing still works with concepts of 'culture', 'illness' and 'disease' that have since been revised. I hope with this book to invite medicinal ethnobotanists to be more open to a phenomenologically-inspired approach that takes seriously people's experience of plants and that focuses on the practices in which people engage when they interact with plants. Medical anthropologists, in light of current trends Beyond the 
Body Proper (Lock and Farquhar 2007), may become more interested in the study of medicinal plants as cultural artefacts, and in their social lives as an aspect of material culture.

SAV: You discussed Thomas Reid's philosophy of 'common sense' and James Gibson's 'ecological psychology of perception' and advocate combining them for the investigation of medical realities. You present them as distinct from empirical and functional knowledge that dominates medicinal ethnobotany. Can you expand on that?

EH: It is really a question why do many medical anthropologists find medicinal ethnobotany so limited, and do not want to engage with it. I think it has to do with its philosophical orientation; ethnobotany appears wedded to an uninteresting kind of empiricism. Medical anthropology has gone a different way. Narrative theory has questioned the idea of a single truth Postmodernism, practice theory, Foucauldian questions of institutional power and more recently the phenomenological twist, the interest in the body, personhood and the self, and the impulse we got from science studies to investigate technologies, materiality and material culture. The two orientations are not easily merged. It seems to me that it is a philosophical problem.

The ethnobotanist Scott Atran talked about common sense as some kind of 'manifestly perceivable empirical fact' (Atran and Medin 2008). For an anthropologist a 'manifestly perceivable empirical fact' does not exist. Scott Atran cited George Moore who cited Thomas Reid, and Reid I found the most carefully formulated and readable author. His philosophy of common sense concerns the self and how it relates the environment. It posits that in certain situations the self can engage with the world in an unmediated way, such that the self and the environment form a continuum. Thomas Reid (1764 [1997]) was at the time arguing against his contemporary David Hume, who in a typical empiricist fashion was very sceptical about the veracity of whatever is mediated through the senses. I thought Reid was interesting as this interrelatedness with the environment until very recently has been dematerialised in medical anthropology. Reid, moreover, has extremely interesting thoughts about the senses and perception, and, in my reading, some of them point in the same direction as Thomas Gibson's Ecological Approach to Visual Perception (Gibson 1979) and the notion of affordances that Gibson developed in that context.
Both authors validate the sensory experiences of the common person in ways that scientific empiricism does not, as the latter takes seriously only expert knowledge. So, there is also a knowledge political edge to my argument. If one implicates the self, and its modes of perception, into the study of what appears commonsensical, this may provide a meeting ground for both disciplines.

SAV: Your fascinating account of the ubiquitous presence of qinghao throughout Chinese materia medica highlights the importance of history of medicine and linguistic interpretations to unravel the changing nature of medicinals as cultural artefacts. What are the implications for ethnobotanical enquiry when ethnomedical remedies are studied as 'cultural artefacts' instead of 'natural herbs'?

EH: It is often said that complementary and alternative medical practitioners work with 'natural herbs'. This is pretty wrong. What is a 'natural herb'? The chemical constitution of the 'herb' depends on the time plants are harvested, the location of where the plants grow, on their preparation and application. In most traditional medicines one mixes plants with other ingredients, like honey, or with other herbs, or one burns them to ashes, dries them in the shade or sun, rolls them into pills or pounds them into powders, Therefore, processed plant materials don't only have different cultural meanings and connotations but also different chemical properties, with different medical effects. Pharmaceuticals as well as the 'natural herbs' are cultural artefacts and have

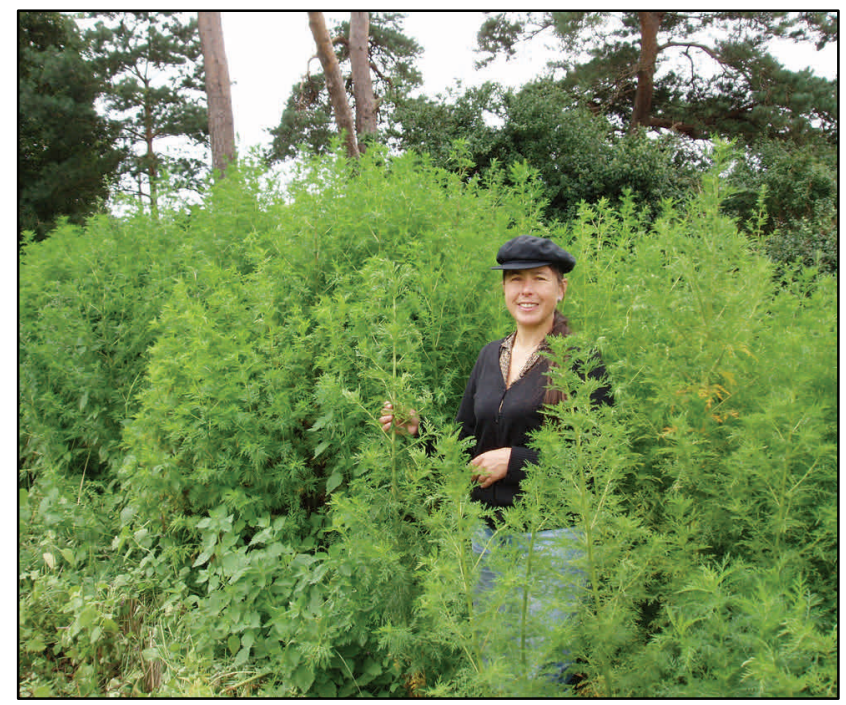

Figure 2. Elisabeth in a field of sweet wormwood, Artemisia annua L. (Asteraceae). 
social lives.

SAV: The discussion on the differences between the cultures of different disciplines goes back quite a while. C.P. Snow in 1959 remarked on the 'two cultures' of modern society - the humanities and sciences (Snow 2001 [1959]). How can we better integrate social and biological sciences for interdisciplinary benefits and enhanced knowledge in the understanding of human sickness and its treatment?

EH: I do not believe in integration through one language. Human beings are born with the ability to speak many languages. The human mind has the capacity to be multi-lingual. In Burkina Faso I was living in a compound visiting my godchild shortly after her birth, and in this compound there were five languages spoken by children under nine. Our knowledge is enhanced by learning the language of different disciplines; learning to frame the problem in different ways and learning to see different perspectives. There are many more than two cultures of modern society: there are enormous differences in the culture of doing science even within the School of Anthropology at Oxford. Within the medical sciences there are differences between geneticists and epidemiologists; the epidemiologists, like anthropologists, emphasize the environmental factors causing disease. And among the geneticists there are the systems biologists who stress the importance of the phenotype when it comes to the transmission of genetic information. It is very important for scholars in the

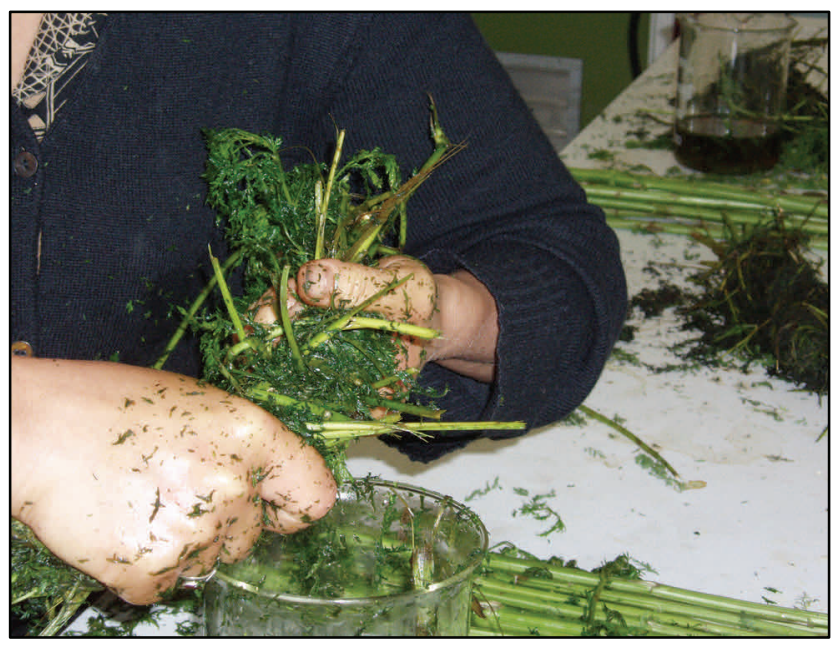

Figure 3. Preparing qinghao by macerating Artemisia annua leaves and squeezing the juice in cold water. Quing hao contains artemisinin which is currently recommended as an antimalarial by $\mathrm{WHO}$. humanities to have an inkling of the natural sciences, and for natural scientists to have one of the social sciences.

SAV: Do you see empiricism, bioculturalism, and neo -Darwinism as part of the same framework?

$\mathbf{E H}$ : Weckerle, Timbul and Blumenshine ask why is it that, worldwide, all seven plant genera that have species producing caffeine (Camellia, Coffea,, Cola, Ilex, Paullinia, Theobroma and Citrus) have been put to use in ritual? It is really amazing because these species are in entirely different botanical families and grow in geographically completely unrelated areas. They are used in meditation rituals, in tea ceremonies; they are chewed, drunk or smoked. What is it between human beings and caffeine? It is a psychotropic drug and awakens the mind. This falls nicely into a biocultural framework of explanation: it is a matter of adaptation. Weckerle and her colleagues mention adaptation and they bring into this discussion human observation of animal behaviour, but they go further in their explanation by pointing to cultural and economic historical changes. I find any kind of research into these phenomena fascinating. What I find problematic is the idea of adaptation as explanation for their existence. Bioculturalist explanations often subsume socio-cultural processes within one species, homo sapiens, into a framework of explanation with which Darwin explained species differentiation over geological, not historical or biographical, time periods. The concept "environment" itself is problematic not only because it implies that it is static. The relationship between the self and other organisms needs to be rethought. Darwinists themselves, like Levins and Lewontin (1985) have long questioned the concept of adaptation.

SAV: Major global challenges like public health, food security, climate change and natural disasters when combined with demographic change at current levels call for ethnobiology and medical anthropology to join forces to deliver benefits to the communities studied and vulnerable communities in general. How can that be achieved?

EH: Applied medical anthropology contributes directly to public health. This book is not about this. It takes seriously cultural history and gives an understanding for the interrelatedness of human beings with the plant world. It tries to get away from focusing on plant chemistry and its medicinal exploitation. It shows how human beings and plants are 
part of one world, entwined in sometimes strikingly similar cultural histories. In the sciences, knowledge is enhanced by a subject-object relationship where the subjective experiences are to be distrusted and the environment is to be objectified; the self has to be detached from the object it studies and a distance is to be produced. There might be another attitude, which arises from a heightened sensitivity to the experiential in human-plant interrelations that are taken for granted. The benefit it might provide for people in these disaster zones is to sharpen their consciousness for these interconnections in everyday life.

\section{References Cited}

Hsu, E. and S. Harris, eds. 2010. Plants, Health, and Healing: On the Interface of Medical Anthropology and Ethnobotany. In the series Epistemologies of Healing, volume 6. Berghahn, Oxford and New York.

Lock, M. and J. Farquhar, eds. 2007. Beyond the Body Proper: Reading the Anthropology of Material Life. Duke University Press, Durham and London.

Atran S. and D. Medin. 2008. The Native Mind and the Cultural Construction of Nature. MIT Press, Cambridge MA.
Reid T. 1764 [1997]. An Inquiry into the Human Mind on the Principles of Common Sense. Ed. Derek R Brookes. Edinburgh University Press, Edinburgh.

Gibson, J. J. 1979. The Ecological Approach to Visual Perception. Houghton Mifflin, Boston.

Snow, C. P. 2001 [1959]. The Two Cultures. Cambridge University Press, London.

Levins, R. and R. C. Lewontin. 1985. The Dialectical Biologist. Harvard University Press, Cambridge, MA.

\section{Biosketch}

Elisabeth Hsu is Professor in Anthropology and Fellow of Green Templeton College, University of Oxford. Her research interests concern Chinese medicine, transmission of knowledge and practice, body and personhood, as well as touch, pain, feelings, emotions, and sensory experience.

Dr. Sofia Anthi Vougioukalou is a Research Associate at the Department of Primary Care and Public Health Sciences at King's College London and an Honorary Research Fellow at the School of Anthropology and Conservation at the University of Kent (UK). 